\title{
A Brief Tour on Exotic Control Objectives in Robotics
}

\author{
Rafael Kelly ${ }^{1}$ and Carmen Monroy ${ }^{2}$ \\ 1. DET-DFA, Division de Fisica Aplicada, CICESE, Carretera Ensenada-Tijuana No. 3918, Zona Playitas, Ensenada, B.C., 22860, \\ Mexico \\ 2. ISEP-Sistema Educativo Estatal, Zona 01, Ensenada, B.C., 22800, Mexico
}

\begin{abstract}
Formulation of control objectives is a key issue in automatic control systems design. Although at first sight the desired goal (control objective) of a control system seems to be a trivial and obvious matter, for effectiveness of some high level robotic tasks, unusual exotic control objectives may be required. This paper presents a review of some exotic control objectives useful in robotics, such as velocity field control objective and range control objective. The paper also proposes a novel confinement control objective. The usefulness of these exotic control objectives may appear in safe robot-human interaction and self-protection of robots against collisions.
\end{abstract}

Keywords: Control objective, robot, robot-human, confinement, range, velocity field, TEFDA (total energy function with damping assignment), exotic.

\section{Introduction to Elements of Automatic Control of Robots}

Robots as a kind of amazing autonomous machines are equipped with digital computer implemented automatic control system (the "robots brain"). So, the effectiveness, applicability and accuracy of robots depend strongly on features of the underlying control system.

It is recognized that many standard robot manipulator applications such as parts positioning/handling, painting, and pick-and-place can be well accomplished by standard well-established control systems such as PID control or compute-torque control [1-3]. Notwithstanding, some other new and more challenging robotic tasks such as safe robot-human interaction, multi-robot cooperation/competition, robot self-protection against auto-collisions, tasks under multi-sensor fusion, and robot tasks under embedded dynamic and unstructured environments are unable to be done by using such standard textbook control systems, hence this paper claims that a broad spectrum for innovation in novel

Corresponding author: Rafael Kelly, Ph.D., professor, research fields: automatic control, robotics, powerful high task-level control system is still open in robotics sheltered under the so-called in this paper as exotic control objectives. This paper is an enhanced version of an early one presented in Ref. [4].

This paper adopts the following definition of robot.

$$
\text { Definition 1-Robot }
$$

"A robot is a reprogrammable, multifunctional, and autonomous amazing animate machine”.

Remark 1: This definition 1 includes both mobile robots as well as robot manipulators.

Remark 2: Although some engineers use the word "autonomous" to refer robots where the computational hardware is close - on board - to them, in this paper the word "autonomous" is more related to a decision making meaning: without neither human intervention nor human assistance during motion or during task execution. Thus, machines under remote control/handling via wireless or umbilical cable communication by a human operator such as drones, telemanipulators, or ROVs (remotely operated underwater vehicles), are not really robots. Exoskeletons and prosthesis are not either considered robots because they are "on-board" human-pilot-operated. 


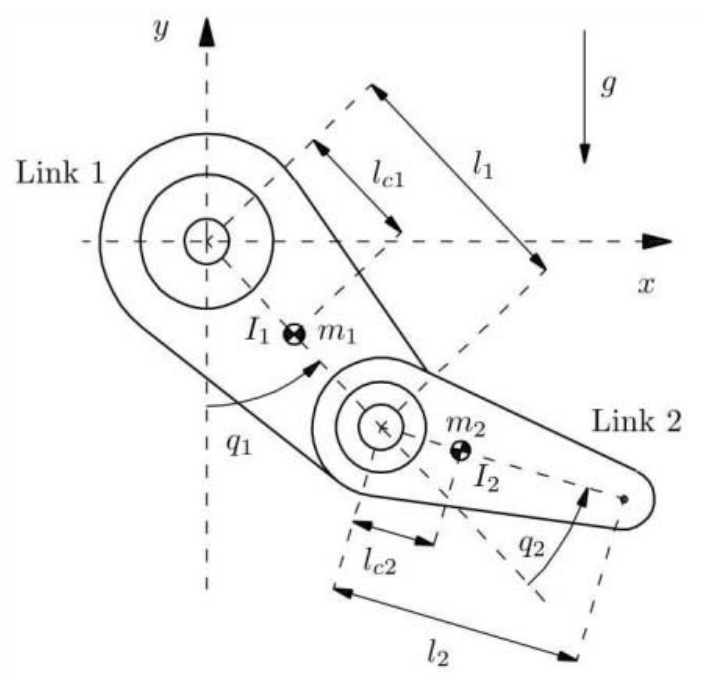

Fig. 1 Sketch of the 2 DOF $\mathcal{R R}$ Planar "Pelican robot" [1].

Through the paper, for $A \subset \mathbb{R}^{n}$ and $p \in \mathbb{R}^{n}$, the distance from a point $p$ to a set $A$ denoted by $\operatorname{dist}(p, A)$ is defined as the smallest distance from point $p$ to any point in $A$; more precisely [5]:

$$
\operatorname{dist}(p, A) \triangleq i n f_{x \in A}\|p-x\|
$$

where $\|\cdot\|$ stands for the Euclidean norm.

For illustration purpose in this paper the $2 \mathrm{DOF} \mathcal{R} \mathcal{R}$ "Pelican robot" [1] shown in Fig. 1 shall be evoked.

\subsection{Control System}

In few colloquial words a control system is an interconnection of components forming a system configuration that will provide a desired behavior. Beyond physical matters, the main conceptual ingredients of a control system are:

- Plant;

- Actuators and sensors;

- Control objective;

- Controller.

Among them the main sine qua non element is the control object or plant defined roughly by:

Plant: The device, physical process, or system to be controlled. Interaction is defined in terms of variables_-“Signals” in engineering jargon; usually $y=z-[6]$ :

(1) Plant controlled/manipulable input $u$;
(2) Plant output $y$ of all sensors;

(3) Plant variable to be controlled $z$. Typically, controlled variable is also a measured one, so $y=z$;

(4) Environmental disturbances $\omega$. Disturbances can be seen as no manipulable/controlled inputs. However, in some cases they may be measured.

\subsection{Plant}

In this paper a plant (physical real world system like a robot, see Fig. 2, or intangible abstract mathematical system to be controlled) is characterized in an abstract way by a 3-tuple $\Sigma_{P}(\mathcal{C}, \mathcal{U}, \mathcal{Y})$ by means of an Input/Output description through the map/operator $\Sigma_{P}$ :

$$
\begin{gathered}
\Sigma_{P}: \mathcal{u} \rightarrow \mathcal{Y} \\
u \mapsto y
\end{gathered}
$$

where

$\mathcal{C}$ is the configuration space (dimension $n$ ); domain of internal variables, e.g., generalized positions or state variables;

$\mathcal{U}$ is the input space (dimension $p$ );

$\mathcal{Y}$ is the output space (dimension $m$ ).

This definition of a plant can include a number of standard robot models (mobile robots as well as robot manipulators) such as [7]:

- geometric models;

- kinematic models;

- differential kinematic models;

- dynamic models.

In the control issue so-called "control of torque-driven robot manipulators in joint space” the robot plant $\Sigma_{P}$ is the joint space dynamic model where $y=\mathcal{C}$, and output $y$ corresponds to the generalized joint positions $q \in \mathcal{C}$, and the input $u \in \mathcal{U}$ is the torque/forces $\tau$ applied at the robot joints.

\subsection{Control Objectives}

Generally speaking, the objective in a control system is:

(1) to make a plant behaves in a desired way;

(2) by manipulating its input u [6]. 


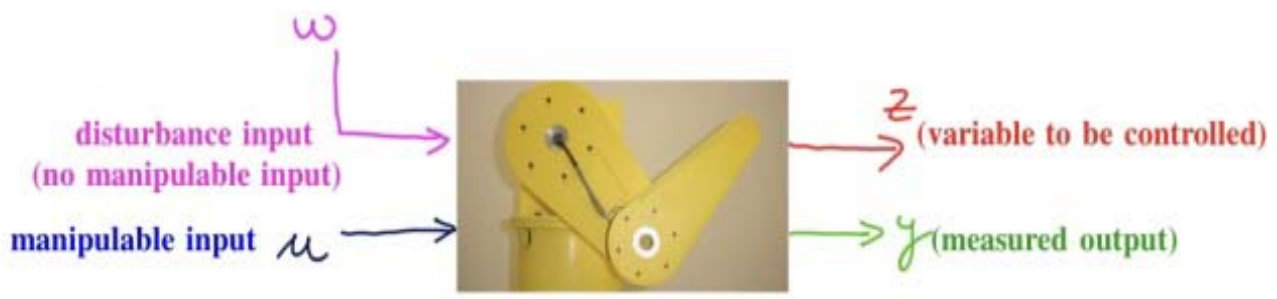

Fig. 2 Plant: Input/Output variables.

Notice that for "plant behavior in a desired way" no explicit allusion to the plant measured output $y$ has been performed, notwithstanding this is usually the case, i.e., to make the plant measured output $y$ or unmeasured variable to be controlled $z$ behaves in a desired way (typically in an asymptotic fashion). But this paper emphasizes that this is not mandatory. In some application such a "plant desired behavior" may be captured by unmeasured plant variables (to be controlled!), say $z$ in the control jargon [6]. Something surprising, it is allowed in automatic control to intend control-force a desired behavior-of unmeasured plant variables $z$ with feedback (closed-loop) or without feedback (open-loop) of measured output ones $y$.

Intuitively, the meaning of the control objective should be fairly obvious to people with some knowledge of automatic control. Nevertheless, intuition has its limitations.

Roughly speaking:

- A control objective is a goal, reason or purpose for which an automatic control system should be implemented.

- A control objective provides a specific target against which is to evaluate the effectiveness of an automatic control system. A control system is said to be effective provided that its control objective is achieved. Many control systems may exist which are able to achieve a given common control objective.

Most of engineering challenges must begin by a clear, precise and unambiguous problem specification together with a wish or objective to be achieved. But, in contrast with standard common sense, the wishes in some engineering applications may be vague and sometimes unclear and ambiguous. This may also occur in control engineering and robotics [4].

Classifications of control objectives may include features as: locally or globally; asymptotic-time or finite-time; e.g., rare but practical control objectives like: finite-time global tracking is also possible.

This paper classifies the control objectives in two groups: standard and exotic.

(1) Standard Control Objectives:

- Regulation: keep controlled variable $z$ close to a constant target value — setpoint, say $r$-;

- Tracking: keep measured variable $y=z$ close to a time-varying target value $y_{d}(t)$, see Fig. 3 .

(2) Exotic Control Objectives:

- Velocity field;

- Range;

- Immobilization;

- Reach;

- Confinement;

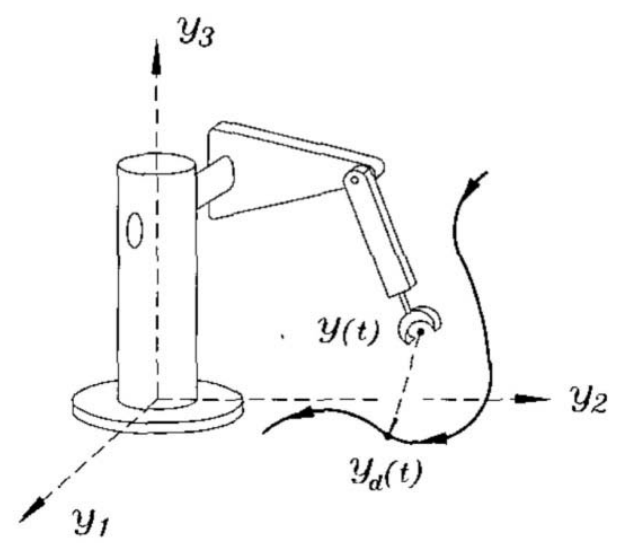

Fig. 3 Standard "Position Tracking" control objective in output space (Cartesian space).

Desired output trajectory: $\boldsymbol{y}_{\boldsymbol{d}}(\boldsymbol{t}) \in \boldsymbol{y}$. 


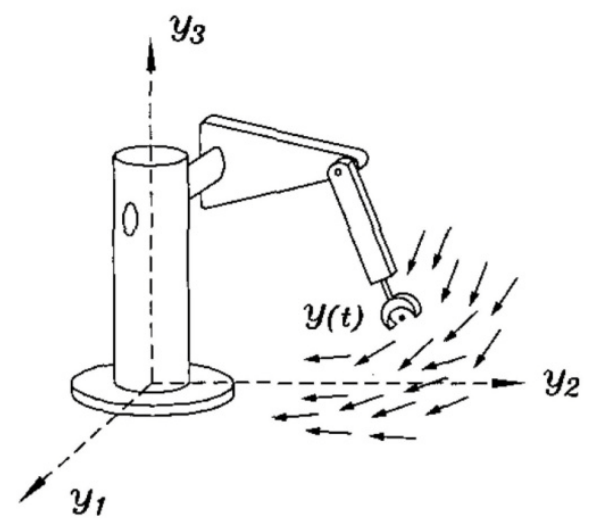

Fig. 4 Velocity field control objective concept $[9,10]$.

- Path;

- TEFDA [12];

- (Ride) Comfort [15, 16].

Some of these exotic control objectives shall be re-called/introduced below.

\section{Velocity Field Control Objective}

Although an original velocity field controller but under a passive approach was first introduced in 1999 by $\mathrm{Li}$ and Horowitz [8], this paper borrows the velocity field control objective definition stated later in Refs. [9, 10] without regard of neither passive requirement nor passive formulation. More precisely:

Definition 2-Velocity Field Control Objective

Given an user defined smooth desired vector field $v(y): \mathcal{Y} \rightarrow \mathcal{T} \mathcal{Y}$, where $\mathcal{T} \mathcal{Y}$ denotes the tangent bundle of $\mathcal{Y}[8,11]$. The velocity field control objective is defined by:

$$
\lim _{t \rightarrow \infty}(v(y(t))-y(t))=0
$$

In this velocity field control objective [10], the desired task to be accomplished by the robot is also coded by means of a smooth velocity vector field $v(y)$ defined in the output space $\mathcal{Y}$ and denoted as a map:

$$
\begin{gathered}
v: \mathcal{Y} \rightarrow \mathcal{T} \mathcal{Y} \\
y \mapsto v(y)
\end{gathered}
$$

This control objective concept is illustrated in Fig. 4 where the robot output $y=z$ is expected to follow flow lines in the velocity field represented by arrows in the output space $y$.

\section{Range Control Objective}

Generally speaking, the regulation control objective in a control system is to make some output, say $y$, to be exactly a desired constant setpoint, say $r$. Although this may be an acceptable (theoretical, academic, classroom or textbook) wish, due to the following arguments, such a wish may be unrealistic or unrealizable:

- Instead of a constant exact value $r$, real control system desired goal may require to keep the output $y$ within a prescribed interval; For example [14]:

- In papermaking the moisture content must be kept between prescribed values.

- Sensors and measurement instruments have always uncertainties in some degree, so it may be unrealizable to wish the output $y$ to get exact precise values; instead, it is more realistic to maintain the output $y$ within a desired range according to sensors and measurement devices accuracy.

Let borrow the following two clever paragraphs from the Janert's book [14]:

(1) "A standard feedback loop is not suitable for maintaining a metric within a range of values; instead, it will try to drive the output metric to the precise value defined by the setpoint $r$."

But in some real world control engineering applications:

(2) "We do not care about tracking a setpoint accurately. Instead, we want to prevent the process output from leaving a specified interval.”

Inspired in above paragraphs, this paper introduces the following novel control objective:

Definition 3-Range Control Objective

Given a desired interval-desired region- $\mathcal{J}_{d} \subset \mathcal{Y}$ the range control objective is defined by:

$$
\lim _{t \rightarrow \infty} \operatorname{dist}\left(y(t), \mathcal{J}_{d}\right)=0
$$

for all $y(0) \in \mathcal{y}$. 


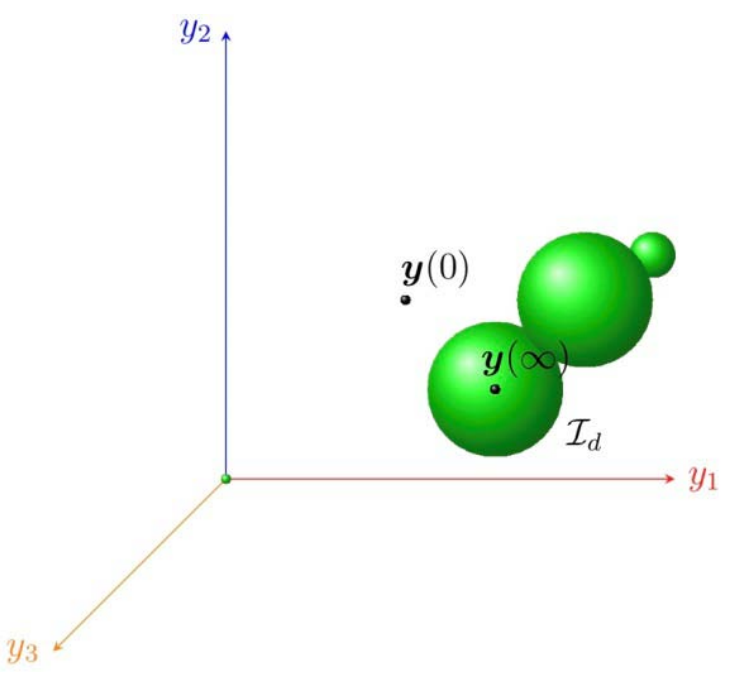

Fig. 5 Range control objective concept.

Desired region $\mathcal{J}_{d}$ in green; $y(\infty) \in \mathcal{J}_{d}$.

The concept of range control objective is illustrated in Fig. 5 where the output $y(t)$ starting from its initial output $y(0)$ is expected to reach as $t \rightarrow \infty$ (with abuse of notation to reach $y(\infty)$ ) the desired interval—region- $\boldsymbol{J}_{\boldsymbol{d}}$.

Remark 3: Although in the range control objective definition above it is not explicitly stated, in this paper it is understood that $\lim _{t \rightarrow \infty} \dot{y}(t)=0$ as well. Instead, an alternative useful and practical option may be: $\lim _{t \rightarrow \infty}\|\dot{y}(t)\| \leq \epsilon_{d}$ for a user defined $\epsilon_{d} \geq 0$. Under this approach, i.e., for a small positive number of $\varepsilon$ this control objective may be applied for sensorless safe robot-human interaction because eventual robot impacts under robot low kinetic energy (due to low robot speed) may reduce undesired human traumatic injuries [17-20]. In the range control objective the intermediate path $y(t)$ between the initial output $y(0)$ and the final one $y(\infty) \in \mathcal{J}_{d}$ does not care.

Remark 4: In case when the desired interval has a unique element, say $y_{d}=\in \mathcal{Y}$, i.e., $\mathfrak{J}_{d}=\boldsymbol{y}_{d}$, then the standard regulation control objective is recovered:

$$
\lim _{t \rightarrow \infty} y(t)=\boldsymbol{y}_{d}
$$

So, in this sense the range control objective can be seen as a generalization of the classic regulation control objective.

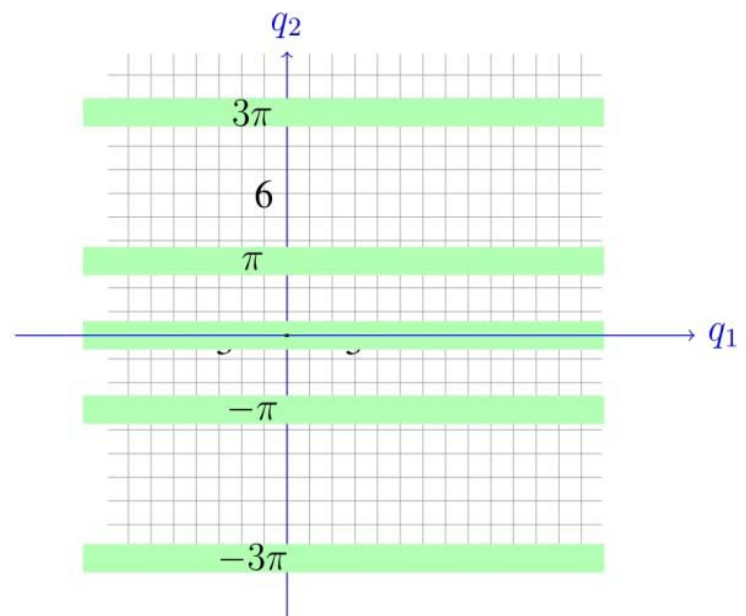

Fig. 6 Example of Range control objective in configuration space $\boldsymbol{y}=\boldsymbol{C}$.

Unfolded 2 DOF arm (desired region) $\mathcal{J}_{d}=\left\{q_{1} \in \mathbb{R}, q_{2} \in\right.$ $-3 \pi,-\pi, 0, \pi, 3 \pi, \ldots\}$ in green.

Example 1: As an academic illustration of the range control objective, let us consider a planar 2 DOF (degrees of freedom) $\mathcal{R} \mathcal{R}$ robot arm like the "pelican" robot described in Ch. 5 of book [1] and shown in Fig. 1. It is wished that: the arm tip reaches one of the distant points on the external border of its 2D Cartesian workspace-circle-(but no specific point is given, it does not care). In other words, the desired robot task is to reach an extended unfolded configuration where both links are collinear (remote link seen as an extension of the close one). This goal-fully extended unfold robot arm-can be attained efficiently by invoking the range control objective with the desired interval defined as:

$$
\mathcal{J}_{d} \triangleq\left\{q_{1} \in \mathbb{R}, q_{2} \in-3 \pi,-\pi, 0, \pi, 3 \pi, \ldots\right\}
$$

which is illustrated as shadow green zones in the configuration space in Fig. 6

\section{Immobilization Control Objective}

This paper introduces the following novel control objective:

\section{Definition 4-Immobilization Control Objective}

For a robot plant (1) the immobilization control objective is defined in this paper as

$$
\lim _{t \rightarrow \infty} \dot{y}(t)=0
$$


for all $\boldsymbol{y}(0) \in \mathcal{Y}$ and $\dot{\boldsymbol{y}}(0) \in \mathcal{T} \mathcal{Y}$.

Although at first glance it seems to be a nonsense or useless control objective in robotics and mechanisms, its utility can be evoked to achieve robust robot emergency stops and docile robot-human interactions, as well as to dominate runaway robots and mechanisms such as out of control robots and spinning satellites catapulted in orbit (no to be confused with the related but different "attitude control" where specification of satellite desired orientation is mandatory). Notice that neither desired output $y_{d}=\in \mathcal{Y}$ nor desired range regions $\mathcal{J}_{d} \subset \mathcal{Y}$ are specified.

\section{Confinement Control Objective}

This paper introduces the following novel control objective:

\section{Definition 5-Confinement Control Objective}

Let a robot be modelled by the plant structure (1). Given a desired settling time $T_{d} \geq 0$ and a desired set $\mathcal{C}_{d} \subset \mathcal{Y}$ called the desired confinement set (Both: $T_{d}$ and $\mathcal{C}_{d}$ are user specified). The confinement control objective is defined here as:

$$
y(t) \in \mathcal{C}_{d} \forall t \geq T_{d} .
$$

In words, the confinement control objective is achieved if the output $y(t)$ reaches at desired settling time $T_{d}$ the desired confinement set $\mathcal{C}_{d}$ and it remains there for all future time $t \geq T_{d}$, see Fig. 7 .

Remark 5: If the initial output $y(0)$ starts into the desired confinement set $\mathcal{C}_{d}$, then the confinement control objective means that the output $y(t)$ will remain into the same desired confinement set $\mathcal{C}_{d}$ forever. Although it is not formally the same concept, $\mathcal{C}_{d}$ may be thought-out as a kind of desired invariant set; the latter is a concept of a proper well defined ordinary differential equation.

Remark 6: A related control objective is the so-called "reach control objective" [13] where entrances to "confinement set" are also specified.

Remark 7: Although different, the confinement control objective may be thought as a kind of

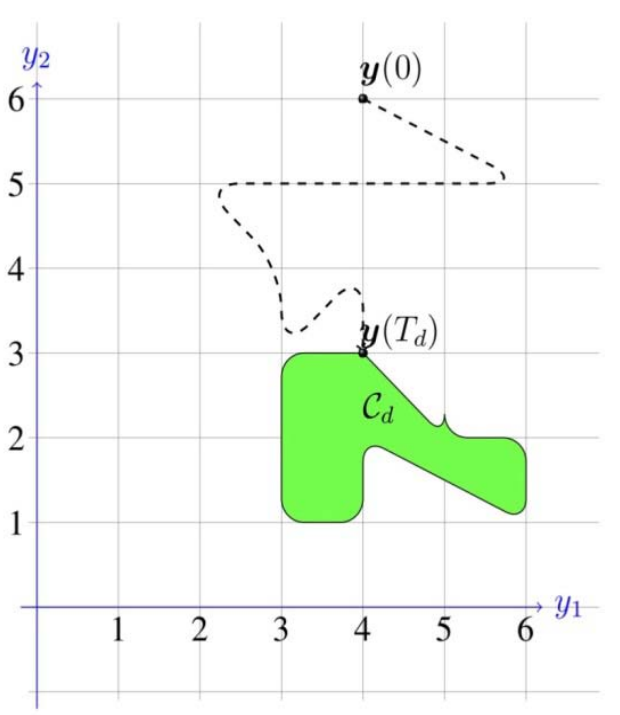

Fig. 7 “Confinement control objective” concept.

Desired confinement region $\mathcal{C}_{d}$ in green color; Trajectory output $y(t)$ in dashed line.

uniformly ultimately boundedness of the output $y$.

Remark 8: By proper selection of the desired confinement region $\mathcal{C}_{d}$ the usefulness of this confinement control objective arises in self-protection of robots by avoiding auto collisions (this is true for both: multi mobile robots and robot manipulators too).

\section{Conclusions}

In this paper a record of some nonstandard control objective concepts like velocity field control and range control with useful applications in robotics have been revisited or introduced. It opens a broad spectrum for potential control problem formulations of new high-level task robot applications like robots interaction with themselves or their environment even humans. These formulations and control design are issues of future researches.

\section{Acknowledgments}

The authors would like to thank the financial support of CONACyT under grants: 166654, 176587, and 631-295.

\section{References}

[1] Kelly, R., Santibañez, V., and Loria, A. 2005. Control of 
Robot Manipulators in Joint Space. London, UK: Springer.

[2] Siciliano, B., Sciavicco, L., Villani, L., and Oriolo, G. 2009. Robotics. Modelling, Planning and Control. London: Springer.

[3] Spong, M. W., Hutchinson, S., and Vidyasagar, M. 2005. Robot Modelling and Control. Wiley.

[4] Kelly, R., and Monroy, C. 2017. "On Exotic Control Objectives in Robotics." Presented at XIX Congreso Mexicano de Robótica COMROB2017, 8-10 de Noviembre de 2017, Mazatlán, Sin., Mexico.

[5] Khalil, H. K. 2002. Nonlinear Systems (3rd ed.). Prentice Hall.

[6] Doyle, J., Francis, B., and Tannenbaum, A. 1990. Feedback Control Theory. Macmillan Publishing Co.

[7] Kelly, R., and Monroy, C. 2014, "Desatando el nudo gordiano del modelado de robots para fines de control: Inspiración en el cordel de Ariadna: Parte I: Rudimentos.” AMRob Journal, Robotics: Theory and Applications 2: (4): 99-106.

[8] Li, P. Y., and Horowitz, R. 1999. "Passive Velocity Field Control of Mechanical Manipulators.” IEEE Trans. on Rob. \& Auto. 15 (4): 751-63.

[9] Moreno, J., and Kelly, R. 2002. “On Manipulator Control via Velocity Fields.” In Proc. of the 15th IFAC World Congress, Barcelona, Spain.

[10] Moreno, J., and Kelly, R. 2003. "Hierarchical Velocity Field Control for Robot Manipulators.” In Proc. 2003 IEEE Int. Conf. on Rob. \& Auto., Taipei, Taiwan, September 14-19, pp. 4374-9.
[11] Murray, R. M., Li, Z., and Sastry, S. S. 1994. A Mathematical Introduction to Robotic Manipulation. Boca Raton, Florida: CRC Press.

[12] Kelly, R. 2015. “Total Energy Function with Damping Assignment (TEFDA): A novel control objective in robotics." In Proc. XVI workshop in information processing and control (RPIC), Cordoba, Argentina, DOI: 10.1109/RPIC.2015.7497057, 6-9 Oct., pp. 1-5.

[13] Helwa, M. K., and Broucke, M. E. 2015. "Flow Functions, Control Flow Functions, and the Reach Control Problem.” Automatica 55: 108-15.

[14] Janert, P. K. 2014. "Feedback Control for Computer Systems.” O’Reilly.

[15] Alaa, M., et al. 2012. "A New Control Strategy of an Electric-Power-Assisted Steering System.” IEEE Transactions on Vehicular Technology 61 (8): 3574-89.

[16] Lee, C. M., et al. 2016. "Ride Comfort of a High-Speed Train through the Structural Upgrade of a Bogie Suspension." Journal of Sound and Vibration 361: 99-107.

[17] Thornton, S. T., and Rex, A. 2013. Modern Physics for Scientists and Engineers (4th ed.). Cengage Learning.

[18] Ericson, C. A. 2005. Hazard Analysis Techniques for Systems Safety. Wile-Interscience.

[19] Dickinson, M. 2004. "Understanding the Mechanism of Injury and Kinetic Forces Involved in Traumatic Injuries.” Emergency Nurse 12: 30-4.

[20] Nayduch, D. 2009. Nurse to Nurse: Trauma Care. Mc Graw Hill Medical. 